\title{
Abraded Surface Properties of Sintered Carbide SPECIMENS BEFORE AND AFTER DEPOSITION OF A THIN LA Yer USING PVD
}

\author{
Bricín David, Průcha Vojtěch \& Kříž Antonín
}
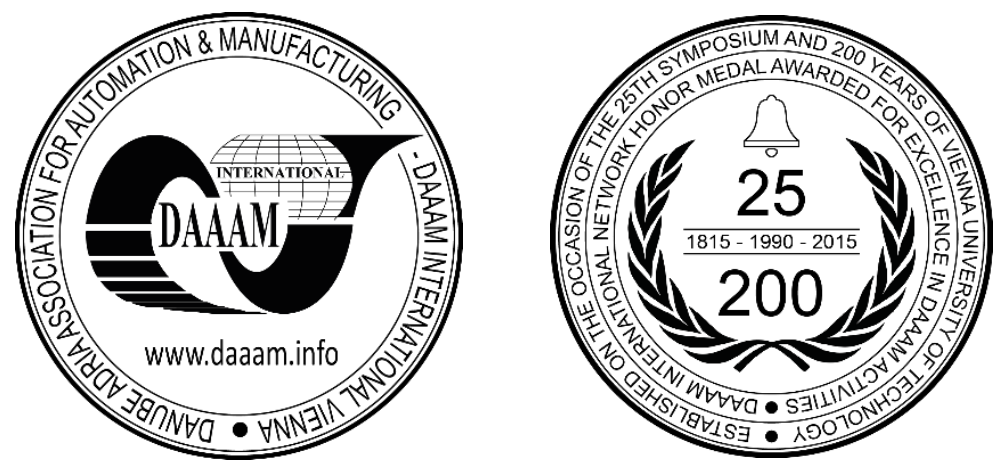

This Publication has to be referred as: Bricin, D[avid]; Prucha, V[ojtech] \& Kriz, A[ntonin] (2017). Abraded Surface Properties of Sintered Carbide Specimens Before and After Deposition of a Thin Layer Using PVD, Proceedings of the 28th DAAAM International Symposium, pp.0937-0943, B. Katalinic (Ed.), Published by DAAAM International, ISBN 978-3-902734-11-2, ISSN 1726-9679, Vienna, Austria

DOI: $10.2507 / 28$ th.daaam.proceedings.130

\begin{abstract}
Two series of samples were produced from sintered carbide WC-Co by using a pulsed laser. Due to the different parameters used for the ablation process, the ablation textures which were created have significantly different properties. From each series of samples, some were selected for deposition of a thin layer by PVD (physical vapour deposition). Metallographic analysis was used to evaluate the properties of the laser treated surface, supplemented by analysis of the chemical composition, hardness, fracture toughness of KIC, tribological properties, and adhesion properties of the prepared samples. The results from the analyses of the ablated surface samples were then compared with the results obtained from the ground surface samples.

By comparing the results, it was found that with the correct parameters set for laser ablation, it is possible to obtain a surface with better abrasion properties for the deposition of the PVD layer on the substrate than with the ground surface.
\end{abstract}

Keywords: Ablation texture; surface treatment; adhesion; laser power; indentation; wear

\section{Introduction}

Sintered carbides are widely used for machining tools because of their mechanical properties, such as hardness and fracture toughness.[6] For some applications, these tools are further deposited with thin PVD or CVD layers. These layers provide a longer tool life because the layer takes over the function of the contact surface between the leaving chips and the face of the tool. In order for the thin layer to fulfil its proper function, it must have good adhesion to the substrate and good tribological properties. These properties are derived both from the chemical composition of the deposited layer and the substrate, and from the surface treatment of the cutting tool by grinding, baking, brushing, and the like. Grinding is mainly used as the technology for surface treatment before the deposition process because residual pressure stresses are initiated in the surface layer of the cutting tool, which is better for prolonging the life of the cutting tool.[1-2] This paper presents some of the results from a study to assess the suitability of surface treatment of cemented carbide by laser and to determine how this surface treatment affects the adhesion and tribological properties of the deposited PVD film. Two series of samples with a laser-treated surface were created, each one made using different parameters. 
For one series of samples, the lowest laser power was used so that the input energy density (Ev) was as low as possible. For the second series of samples, a completely opposite approach was chosen, using the highest possible laser power, and the input value of energy density was approximately 100x higher than in the first series. A comparative series of samples with ground surfaces was then made.

Metallographic analysis using a TESCAN VECTRA electron microscope, supplemented by hardness, fracture toughness and chemical composition analysis using an EDX analyser was used to evaluate the properties of the lasertreated surfaces. The Scratch Test, Pin-on-Disc Test and Mercedes Test were used to evaluate the properties of the deposited thin films.

\section{Results and discusion}

\subsection{Metallographic evaluation of the samples}

The first analysis was the visual assessment of the condition of the treated surface. This evaluation was performed using a TESCAN VECTRA electron microscope, as shown in the photographs below (Fig. 1), which document the samples' surface conditions after laser ablation and grinding.
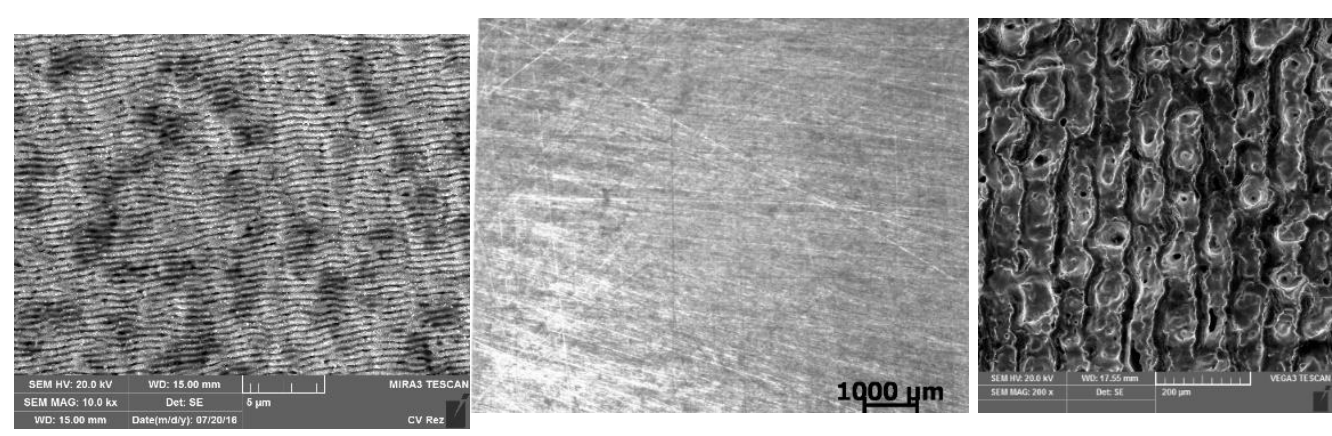

Fig. 1. Samples of treated surfaces. Left: sample surface treated with low energy density of laser. Centre: sample surface with ground surface. Right: sample surface treated with high energy density.

From Fig. 1 it can be seen that the modified surfaces of the samples differ significantly due to the different production parameters. The centre photograph shows typical wear of the surface caused by the grinding wheel. Since the tracks produced go in different directions, it is likely that multi-directional grinding was used to grind the sample surface. By comparing the left and the right images, it can be seen that the high and low energy densities produced a change in the surface layer of the treated cemented carbide. The structure created using low energy density was considerably finer than the structure created by the high energy density. It also shows that the resulting texture has a certain spatial orientation. This orientation was dependent on the direction of the laser movement.

Other differences detected by the electron microscope were differences in chemical composition. Before surface treatment, the chemical composition of each sample was determined by EDX analysis. This chemical composition corresponded to the material supplier's datasheets. This analysis was repeated after the surface treatment of the samples. Comparing the results of the EDX analysis before and after the surface treatment, it was found that all of the samples tested showed a decrease in $(\mathrm{Co})$ in the surface layer of the samples, see Table 1.

\begin{tabular}{|l|l|l|l|c|l|l|l|}
\hline & $\mathrm{W}+\mathrm{C}$ & $\mathrm{Co}$ & $\mathrm{O}$ & $\begin{array}{c}\text { Surface } \\
\text { modification }\end{array}$ & $\mathrm{W}+\mathrm{C}$ & Co & $\mathrm{O}$ \\
\hline $\begin{array}{l}\text { Sample Before } \\
\text { surface treatment }\end{array}$ & $94 \pm 0,076$ & $6 \pm 0,076$ & - & Laser with low Ev & 91.4 & 5.6 & 3 \\
\cline { 4 - 8 } & & & & Grinding & 92.2 & 5 & 2.8 \\
\cline { 3 - 8 } & & Laser with high Ev & 95.5 & 2.4 & 2.1 \\
\hline
\end{tabular}

Table 1. Chemical composition of the samples with treated surfaces detected by EDX analysis.

All samples showed slight surface oxidation during surface treatment due to the fact that no inert protective atmosphere was used in the process. Binder Co losses were caused by the different sets of parameters and technologies which were used for surface treatment of the samples. The use of higher energy density Ev led to a more pronounced loss of binder, resulting in the formation of a brittle surface layer with a thickness of more than 50 microns. The low toughness of the layer was also manifested by the cracking of the layer due to large residual heat stresses. The low tenacity of this layer was also demonstrated in further experiments. There was a loss of binder on the ground surface due to leaching, which occurred during grinding of the sintered carbide. The lowest loss of the binder was detected on the laser-treated 
samples with low energy density Ev. This meant that the layer was tougher than the other samples with laser modified surfaces. The thickness of this affected layer was only a few microns.

The Vickers hardness test at a load of $30 \mathrm{kgf}$ (HV30) was used to show the fragile behaviour of the formed surface layers. See Fig. 2.
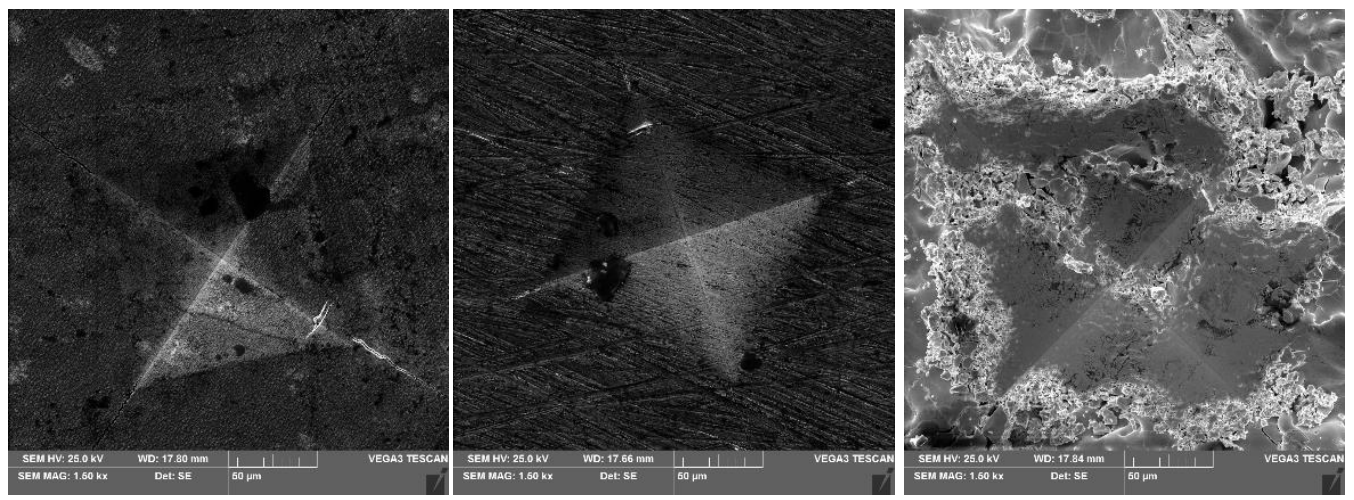

Fig. 2. Indentations created during hardness measurement. For samples with ground surfaces (centre) and samples with surfaces treated by laser with low energy density (left), cracks were found in the corners of the imprints. The shape of indentations was regular. When measuring surface hardness of samples with surface ablation using high energy density Ev (right), the substrate dropped and the shape of the imprints was uneven.

It can be seen from Fig. 2 that when applying a high energy density, the surface layer of the sample was brittle. This embrittlement is caused by a significant loss of binder from this layer by evaporation. This occurs because its evaporation temperature was reached during the ablation process. Due to the decay of the brittle surface layer into fragments, it was not possible to precisely determine its hardness. The hardness measurement was further supplemented by the Palmquist fracture toughness calculation, see Equation 1 and Table 2. However, this measurement was only possible for samples of ground and laser-treated samples with low energy density Ev. Other types of samples did not initiate cracks at the corners of the imprints. This was probably because the applied load caused dropping and breakage of the brittle layer under the indenter so that the energy to create these cracks was used for disruption of the brittle layer, meaning that cracks did not initiate.

$$
\begin{aligned}
& \text { [4] } W_{k}=A \cdot \sqrt{H V} \cdot \sqrt{W_{g}}\left[M N \cdot m^{-\frac{3}{2}}\right] \\
& \text { [4] } W_{g}=\frac{P}{T}\left[N \cdot \mathrm{mm}^{-1}\right]
\end{aligned}
$$

Where:

A ... constant, HV ... hardness according to Vickers HV (30), P ... imprinting load (kgf), T, total length of cracks produced $(\mathrm{mm})$

\begin{tabular}{|c|c|c|}
\hline \multicolumn{2}{|c|}{ Vickers HV30 surface hardness and calculated fracture toughness values Kic } \\
\hline Type of sample & Hardness HV (30) & Kic (Palmquist) \\
\hline Samples with ground surface & $1700 \pm 38.5$ & $12.4 \pm 0.52$ \\
\hline $\begin{array}{c}\text { Samples with laser treated surface } \\
\text { using low energy density Ev }\end{array}$ & $1690 \pm 19.3$ & $9.7 \pm 0.32$ \\
\hline $\begin{array}{c}\text { Samples with laser treated surface } \\
\text { using high energy density Ev }\end{array}$ & - & - \\
\hline
\end{tabular}

Table 2. Calculated fracture toughness and HV30 hardness

The behaviour of the treated sample surfaces was further evaluated using a Pin on Disc tribological test, where the volume loss of material $(\mathrm{mm} 3)$ at the point of contact where the indenter compressed the rotating surface of the test sample was evaluated. [3, 8] This test was further used to evaluate the properties of the deposited PVD layer. For samples which were deposited by a PVD thin layer, the Mercedes Test and Scratch test were used to evaluate the cohesive-adhesive behaviour of the deposited PVD thin film on the modified surfaces of the samples. 


\subsection{Tribological behaviour of the treated specimens}

For the Pin on Disc Test, a $3 \mathrm{~mm}$ diameter carbide ball was forced into the rotating surface of the specimens with a constant force of 10 Newtons. After 20000 cycles, tribological traces were formed on the sample surfaces, see Fig. 3.

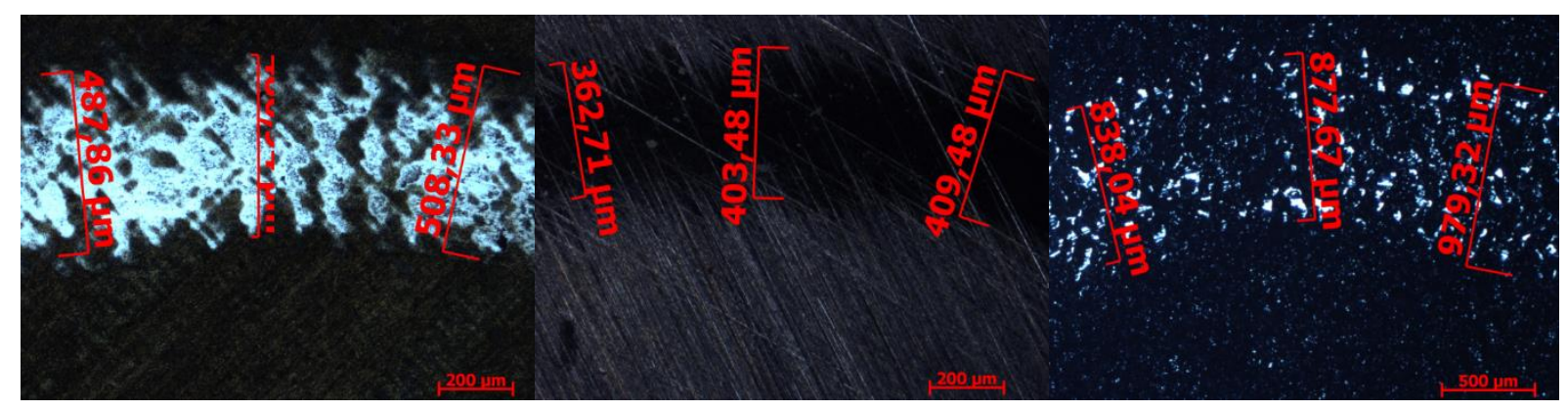

Fig. 3. Differences between tribological traces. Left: tribological trace created on the sample surface treated by laser with low density of input energy $\left(\mathrm{E}_{\mathrm{v}}\right)$. Centre: tribological trace created on sample surface treated by grinding. Right: tribological trace created on sample surface treated by laser with high density of input energy $\left(\mathrm{E}_{\mathrm{v}}\right)$.

Tribological traces were then measured at four different locations. The volume loss of the material was then calculated according to Equation 2 below. Fig. 4 shows a comparison of the volume of material removed from the treated samples surface layers before and after deposition by thin layer PVD.

[7] $\quad V=\frac{\pi(\text { wear track radius, } \mathrm{mm})\left(\text { track width, } \mathrm{mm}^{3}\right)}{6(\text { sphere radius }, \mathrm{mm})}\left[\mathrm{mm}^{3}\right]$

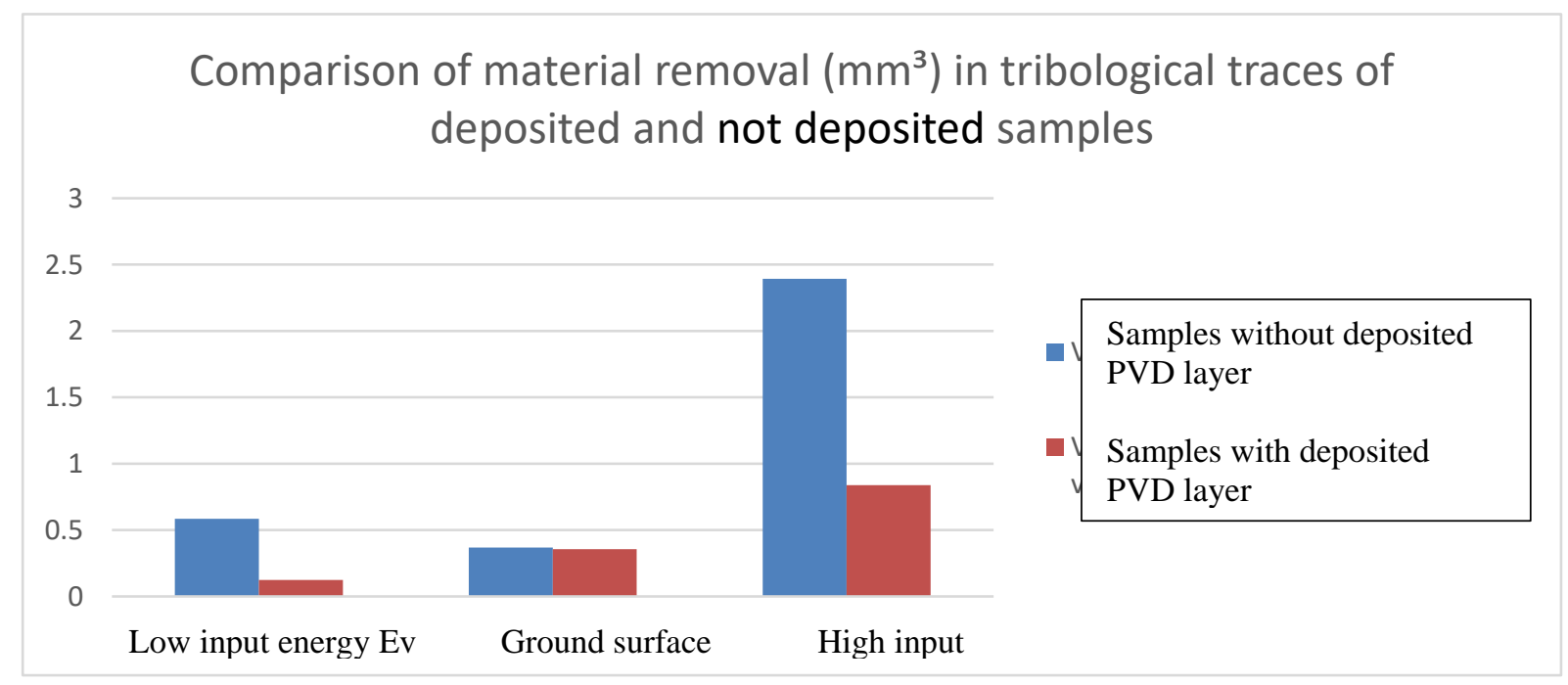

Fig. 4. Comparison of the volume of material removed during Pin on Disc tribological test.

From Fig. 4 it can be seen that the smallest material volume removed was recorded in the samples with ground surfaces prior to the deposition of the thin film PVD on the treated sample surface. This is probably due to the fact that residual pressure stresses were initiated during grinding. These stresses are better for the treated surface layer because they have a tendency to close any cracks which may form at places where the surface of the ground samples is damaged. For samples with a laser-ablated surface treatment, residual stresses are created in the sample surfaces. These types of residual stresses tend to increase cracking which may form at the places where the surface of the laser ablated samples is damaged. Therefore the volume of material removed from the treated surface layer of the ground samples is lower than for the samples with laser ablated surfaces. Another possible reason why the material removal rate was lower for samples with ground surfaces may be the lower surface roughness of these samples. Due to the smoother surface, without extreme rises and drops in the roughness profile, there is no intense tearing of the material from the sample surfaces during their contact with the indenter during the Pin on Disc test. This is another possible reason why the amount of the removed material was smaller for the ground samples than for the samples with laser ablated surfaces. The largest volume loss of material was recorded in samples with laser treated surfaces using high energy density (Ev). This is associated with the brittleness of the layer thus created, which was confirmed in previous experiments. 
After deposition of a thin layer by PVD, there was a decrease in material removal from the surface of the samples. The most noticeable decrease of removed material was observed in samples with surfaces treated by laser. This is probably because surface wrinkling occurred during this technological operation which increases the contact surface between the thin layer and the laser treated substrate. This meant that the adhesion of the deposited thin layer was higher and the material removal was less than for samples with ground surfaces. A reduction in the volume of removed material was calculated for samples with surface treatment by laser using high energy density, but due to the brittleness of this layer, the amount of removed material was still higher than measured on the other samples. In the case of the ground samples, after the deposition of a thin layer by PVD, there was also a reduction of the material removal in the tribological trace, but this decrease is minimal compared with the other samples. The reduction of the volume of material removed for samples with laser treated surfaces using low energy density after thin layer PVD is interesting from a technological point of view, as described above.

\subsection{Adhesive-cohesive behaviour of samples deposited using thin layer PVD}

The adhesive-cohesive behaviour of the deposited thin films was evaluated using the Mercedes test.[5-9] In this test, a Rockwell Cone is pressed into the surface of a specimen with a force of $1500 \mathrm{~N}$. The indenter induces stresses at the edges of the indentation to create tension, which then leads to cracking and peeling of the PVD layer. A Zeiss Observer $\mathrm{Z} 1 \mathrm{~m}$ light microscope and a comparison table (Table 3) were used to evaluate the state of the indentation interfaces.
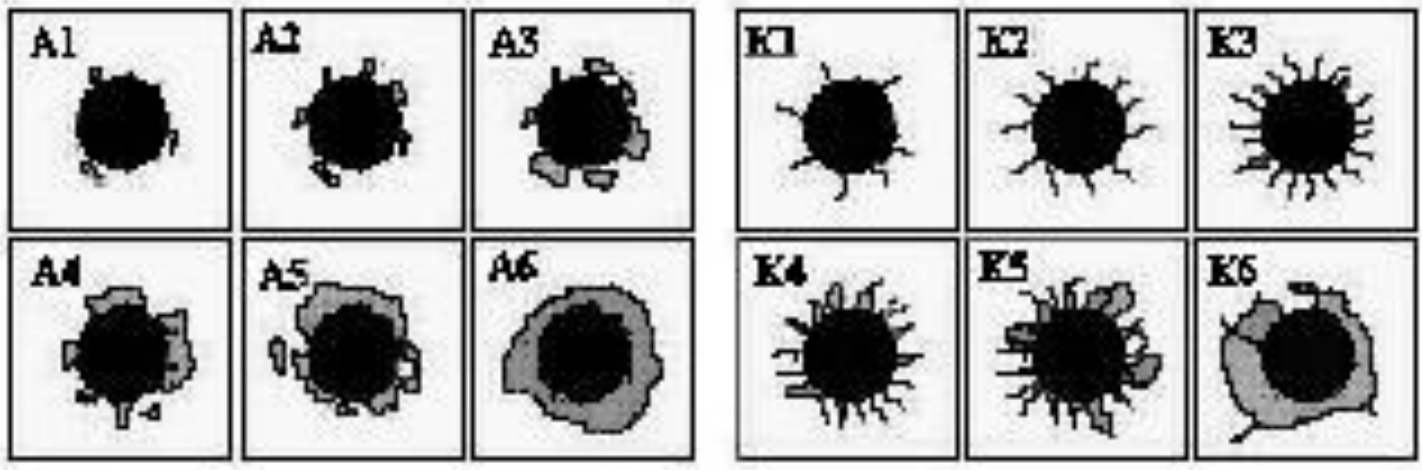

Table 3. Comparison card used to evaluate the cohesive-adhesive behaviour of a pair of thin-layer substrates.[5]

A deposited layer is considered to have satisfactory adhesive properties (A) and cohesive properties (K) with values 1-3. At higher values, the adhesion or cohesion of the layer to the substrate is considered unsuitable, see Table 3.

Fig. 5 shows photographs of the interface of the imprint.
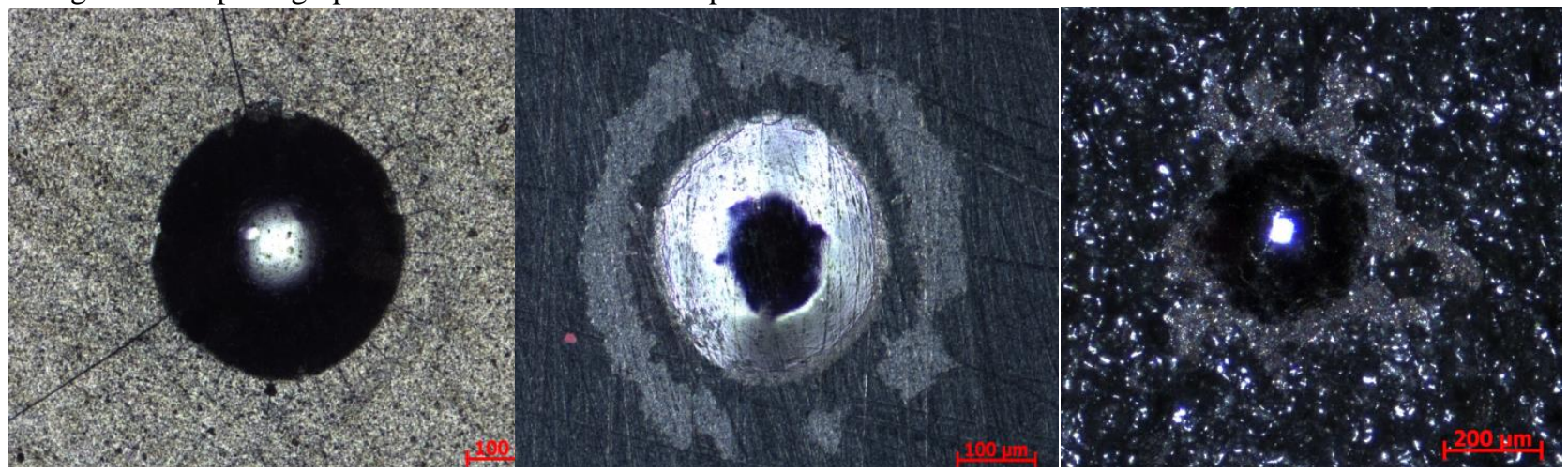

Fig. 5. Sample surface condition after Mercedes test. Left: indentation edge on sample with laser surface treatment using low density input energy (Ev). Centre: indentation edge on sample with ground surface. Right: indentation edge on sample with laser surface treatment using high density input energy (Ev).

It can be seen from Fig. 5 that for samples with ground surfaces and samples with laser treated surfaces using high density input energy there was a tearing off of the deposited thin layer from the substrate surface. This indicates that the adhesion of the layer to the substrate is inadequate. For samples with surfaces treated by laser using low density input energy (Ev) cohesive cracks were found (see Fig. 5, left) at the edge of the indentation. This type of cohesive damage was not as significant as that observed in the other samples. Therefore the cohesive behaviour of all the series of all the samples was evaluated as similar and satisfactory. 
For sample surfaces with a deposited thin layer, a Scratch test was also performed.[5, 8] The results of this test are, however, only indicative, because the surfaces of the samples were too rough after their laser treatment. This caused the indenter, in its straight forward motion, to copy the surface roughness of the specimens and jump from them, thus disturbing the acoustic emission signal.

The traces created during this test could then only be evaluated by using an electron scanning microscope, which allows EDX analysis of the chemical composition of the treated surface layer. This made it possible to find the places where the tearing of the thin layer out of the substrate occurred.

Critical loads Ls $(\mathrm{N})$ were then deducted at the places where the tearing started, which signify the adhesion of the PVD layer against the substrate. In this case it was possible to find the place where tearing started due to the increased percentage of tungsten detected by the EDX analyser. Traces which were created during the Scratch Test are shown in Fig. 6.

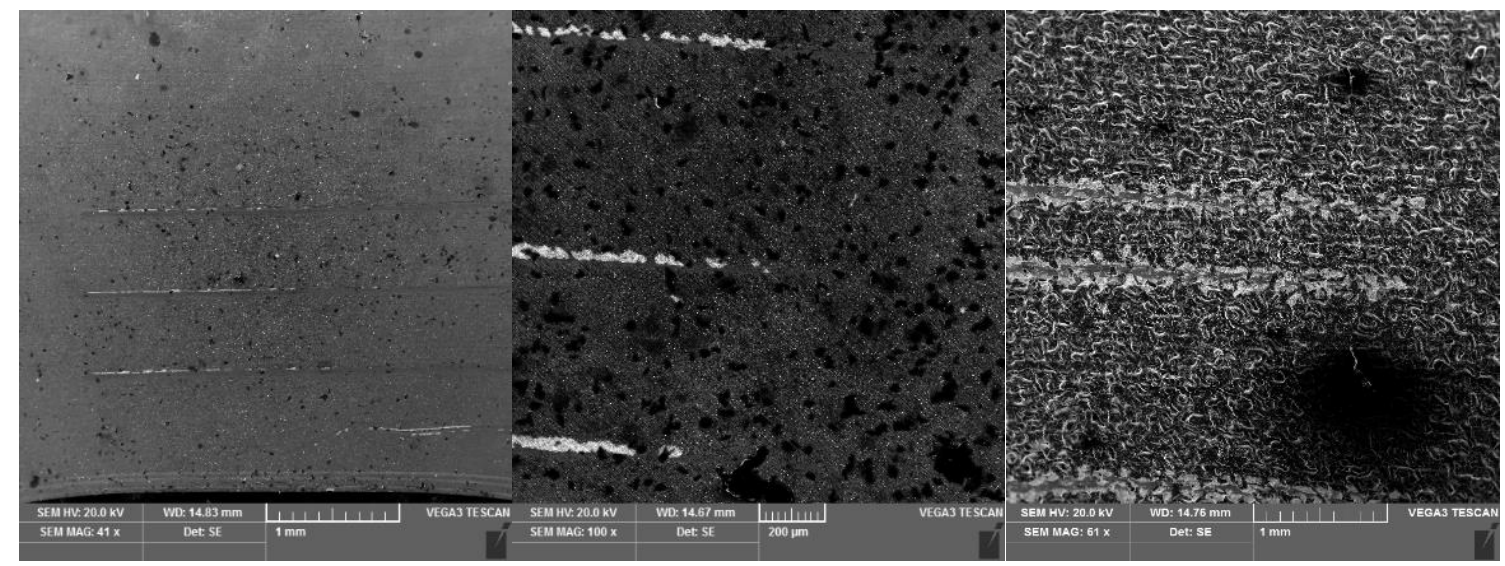

Fig. 6. Indentation tracks created during the scratch test. Left: indentation tracks on sample with laser surface treatment using low density input energy (Ev). Centre: indentation tracks on sample with ground surface. Right: indentation tracks on sample with laser surface treatment using high density input energy (Ev).

\begin{tabular}{|c|c|}
\hline Sample type & Critical load value Ls (N) \\
\hline Low energy Ev & 46 \\
\hline Ground surface & 9 \\
\hline High energy EV & 12 \\
\hline
\end{tabular}

Table 4. Measured Critical Load Values Ls.

From the results given above, it was found that the highest resistance to tearing of the layer was shown by the thin layer deposited on the surface which was treated with a laser using low energy density Ev. For samples with ground surfaces and surfaces treated with a laser using high density input energy, lower resistance to tearing of the deposited thin layer from the substrate was measured. These results confirm the results of the Mercedes test.

\section{Conclusion}

Our results show that we can achieve better adhesion of a PVD layer to a sintered carbide substrate with the correct setting of laser ablation parameters, than for the deposition of a PVD layer on a ground surface. With the correct parameters of laser treatment for sample surfaces with low input energy density, it was possible to achieve the appropriate roughness, i.e. the wrinkling of the surfaces of the samples.

The sample surfaces had good toughness, which differed from the sample surfaces prepared by laser using high density energy input, which was brittle. After deposition by thin layer PVD, other experiments showed that samples with surfaces treated with low input energy density had better tribological properties and adhesion to the substrate.

In this case, the abrasion wear of the laser treated surface of the samples was reduced by about $35 \%$ when using low input energy density, compared to samples treated by grinding. Further experiments will focus on practical applications of the wrinkling created in the machining process.

\section{Acknowledgement}

This article was made possible by the funding for the SGS-2015-016 project "Analysis of Surfaces of Structural Details and Tools by Surface Integrity Method, and Impacts on End-Use Properties”. 


\section{References}

[1] Denkena B. B et al., Residual Stress Distribution in PVD-Coated Carbide, Leibniz Universität Hannover, Germany, Institute of Production Engineering and Machine Tools

[2] Hegeman J. et al., Grinding of WC-Co hard-metals, University of Groningen, Laboratory of Applied Physics, Materials Science Center and The Netherlands Institute for Metals Research.

[3] Hájek J. (2007).Laboratory and practical tests on systems with thin layers used in cutting applications. Ph.D. thesis. UWB in Pilsen. Faculty of Mechanical Engineering, Department of Material Science and Technology. Pilsen 2007.

[4] Janoušek A. (2014). Properties and Cutting Tool Applications of Sintered Carbides. Master thesis. UWB in Pilsen. Faculty of Mechanical Engineering, Department of Material Science and Technology. Pilsen 2014.

[5] Sosnová M. (2007). Relationship of methods, which evaluate the adhesive cohesive behaviour of systems with thin layers. Ph.D. thesis. UWB in Pilsen. Faculty of Mechanical Engineering, Department of Material Science and Technology. Pilsen 2007.

[6] Špirit Z. (2013). Selected Properties and cutting tools applications of sintered caribides. Master thesis. UWB in Pilsen. Faculty of Mechanical Engineering, Department of Material Science and Technology. Pilsen 2013.

[7] ASTM G99 - 17, Standard Test Method for Wear Testing with a Pin-on-Disk Apparatus, (2017) ASTM International, 1. 1. 2017, Available from: https://www.astm.org/Standards/G99. Cited: 7. 8. 2017.

[8] Valova, M. \& Scuhanek, J. (2011). Evaluation of Tribilogical Characteristics of Duplex Coated 31CrMoV9 Steel, Annals of DAAAM for 2011 \& Proceedings of the 22nd International DAAAM Symposium, 23-26th November 2011, Vienna, Austria, Volume 22, No. 1, ISSN 1726-9679, ISBN 978-3-901509-83-4, Katalinic, B. (Ed.), pp. 06350636, Published by DAAAM International Vienna, Vienna.

[9] Lind, L., Peetsalu, P., Põdra, P., Adoberg, E., Veinthal, R. and Kulu, P. (2010). Description of punch wear mechanism during fine blanking process. In Proc. 7th International Conference DAAAM Baltic Industrial Engineering. Tallinn. 